\title{
Análisis comparativo de la información política publicada en Twitter por los principales medios impresos y digitales en torno a las elecciones generales del $10 \mathrm{~N}$ de 2019
} Comparative analysis of the political information published on Twitter by the main print and digital media around the general elections of $10 \mathrm{~N} 2019$

Javier J. Amores

Universidad de Salamanca

Maximiliano Frías-Vázquez

Universidad de Salamanca

Patricia Sánchez-Holgado Universidad de Salamanca

David Blanco-Herrero Universidad de Salamanca

\section{Referencia de este artículo}

Amores, Javier J.; Frías-Vázquez, Maximiliano; Sánchez-Holgado, Patricia y BlancoHerrero, David (2022). Análisis comparativo de la información política publicada en Twitter por los principales medios impresos y digitales en torno a las elecciones generales del 10N de 2019. En: adComunica. Revista Científica de Estrategias, Tendencias e Innovación en Comunicación, $\mathrm{n}^{0}$ 23. Castellón de la Plana: Departamento de Ciencias de la Comunicación de la Universitat Jaume I, 301-328. DOI: http:// dx.doi.org/10.6035/adcomunica.6274

\section{Palabras clave}

Twitter; medios; prensa; elecciones generales; comunicación política; partidos políticos. 


\title{
Keywords
}

Twitter; Media; Press; General Elections; Political Communication; Political Parties

\section{Resumen}

Los medios informativos, tanto los nativos digitales como los todavía impresos, son cada vez más activos en redes sociales, ya que permiten una interacción real con sus lectores, ahora también prosumidores. Los internautas siguen informándose mayoritariamente de la actualidad política a través de las cuentas de esos medios. En este contexto sorprende que, aunque muchos autores analizan la actividad de los políticos en Twitter, son pocos los que estudian los usos que hacen de esta red los propios medios a la hora de cubrir eventos políticos. Este es el objetivo del presente trabajo, en el cual se analizan los tuits e interacciones de los cinco principales medios impresos y digitales españoles en torno a las Elecciones Generales del 10N de 2019. Para ello, se descargaron todos los tuits publicados o retuiteados por las cuentas oficiales de los principales medios impresos y digitales en España las dos semanas antes y después del 10N para analizar la atención que prestaban a la información política. Los resultados confirman que los medios impresos son los que presentan una mayor actividad en sus cuentas de Twitter aunque los medios digitales prestan mayor atención a la información política, y generan mayor interacción. De los partidos políticos analizados, PSOE y VOX son los que tienen mayor presencia en la muestra a nivel general. Los dos partidos que finalmente formaron gobierno - PSOE y UP - vieron incrementada la cantidad de tuits que les mencionaban tras las elecciones, mientras que PP y VOX experimentaron el fenómeno contrario. Las interacciones fueron mayores tras la jornada electoral, tanto a nivel general como en los tuits de contenido político.

\begin{abstract}
News media, both digital natives and printed newspapers, are increasingly active on social networks, which allow real interaction with their readers, now also prosumers. However, the accounts of these media continue to be the ones through which users mostly find out about current political news. In this context, it is surprising that, although many authors analyse the activity of politicians on Twitter, few study the way the media uses this network when covering political events. This is the objective of the present work, which has been achieved by analysing the tweets and interactions of the five main Spanish print and digital media around the $10 \mathrm{~N}$ General Elections. For that, we downloaded all the tweets posted or retweeted by the official accounts of the main printed and digital media in Spain two weeks before and after the $10 \mathrm{~N}$ to analyse the attention they paid to political information. In summary, the results confirm that the print media are the ones with the highest activity on their Twitter accounts, although the digital media pay more attention to
\end{abstract}


political information and generate the most interaction. Of the analysed political parties, PSOE and VOX are the ones with the greatest presence in the sample at a general level. The two parties that finally formed the government - PSOE and $\mathrm{UP}$ - experimented an increase in the number of tweets that mentioned them after the elections, while PP and VOX experienced the opposite phenomenon. Interactions were higher after the election, both at a general level and in tweets with political content.

\section{Autores}

Javier J. Amores [javieramores@usal.es] es personal investigador miembro del Observatorio de los Contenidos Audiovisuales (OCA). Graduado en Comunicación Audiovisual y Master en Investigación en Comunicación Audiovisual con Premio Extraordinario por la Universidad de Salamanca, en la actualidad desarrolla su tesis doctoral en la misma Universidad, con el apoyo financiero de la Junta de Castilla y León y el Fondo Social Europeo.

Maximiliano Frías-Vázquez [maxfrias@usal.es] es personal investigador miembro del Observatorio de los Contenidos Audiovisuales (OCA). Licenciado en Ciencias de la Comunicación por la Universidad La Salle (México) y Máster en Investigación en Comunicación Audiovisual por la Universidad de Salamanca, en la actualidad desarrolla su tesis doctoral en la misma Universidad.

Patricia Sánchez-Holgado [patriciasanc@usal.es] es Doctora en Comunicación por la Universidad de Salamanca, miembro del Observatorio de Contenidos Audiovisuales (OCA). Directora de Comunicación e Innovación de la agencia de publicidad tthegap y responsable del Laboratorio de Investigación. Profesora Asociada de la Universidad Nebrija de Madrid y de la Universidad Pontificia de Salamanca.

David Blanco-Herrero [david.blanco.herrero@usal.es] es personal investigador miembro del Observatorio de los Contenidos Audiovisuales (OCA). Graduado en Periodismo por la Universidad a Distancia de Madrid, graduado en Dirección de Empresas por la Universidad de León, y Máster en Comunicación Audiovisual por la Universidad de Salamanca. En la actualidad realiza su tesis doctoral en la Universidad de Salamanca con una beca FPU. 


\section{Introducción}

La integración de las redes sociales en los flujos de comunicación e información ha supuesto importantes cambios en las relaciones que tienen lugar entre las audiencias y los grandes medios de comunicación (Sáez-Trumper, 2011), pasando de un modelo unidireccional y de masas, a uno en el que todos los actores pueden participar y verse implicados de igual manera (Carlson, 2018). Como Castells (2010) señalaba ya antes de la gran penetración de las redes sociales, estas han facilitado la integración de los ciudadanos y colectivos sociales a los procesos informativos que influencian la opinión pública. No es extraño, en este sentido, que diversos autores aludan a la "ciberdemocracia” (Gil-Ramírez y Gómez de Travesedo-Rojas, 2021). Esto ha hecho que los medios de masas hayan perdido su capacidad monopolística en la construcción de la opinión pública, ya que ahora cualquier usuario puede publicar información y contenidos, que pueden llegar a tener un impacto global (Calvo-Rubio, 2017). Y de todos esos medios sociales, uno de los más analizados por la academia en su condición de herramienta informativa es Twitter (Hermida, 2013; López Meri, 2015a). Esto se debe a que, a pesar de no ser la red social más usada en España, es considerada la que mayor peso tiene dentro del sector periodístico (Bruns y Burgess, 2012) y la más relevante y usada por profesionales de información, así como por políticos e instituciones (Rodríguez y Ureña, 2011; Campos-Domínguez, 2017).

$\mathrm{Al}$ abordar esta red social como herramienta para la comunicación política, el foco ha estado puesto frecuentemente en el uso que de ella hacen los partidos o líderes políticos (Suau-Gomila, 2020; Marín Dueñas y Díaz Guerra, 2016; López-García, 2016; Jivkova-Semova, Requeijo-Rey y Padilla-Castillo, 2017), y especialmente cuando la atención se centra en los contextos que rodean a las campañas y jornadas electorales (López Meri, Marcos-García y Casero-Ripollés, 2017; García-Ortega y Zugasti-Azagra, 2018). Sin embargo, este trabajo busca profundizar en la utilización de Twitter como herramienta informativa no por parte de los políticos, sino por los medios de información españoles, a la hora de cubrir la actualidad política en tiempos electorales, ampliando el trabajo previamente publicado por BlancoHerrero et al. (2020), en el que se exploraba la información política publicada en Twitter por los principales medios en el período previo a estas elecciones. Y es que, aunque las redes han permitido pasar de una comunicación de masas unidireccional a una red de usuarios que interactúan con la información (Carlson, 2018), los propios medios de comunicación de masas y sus profesionales, ya adaptados al mundo digital, siguen asumiendo ahora el rol de gatekeepers en estos nuevos medios sociales (McElroy, 2013), y esto ocurre de manera especial en Twitter, red social informativa por excelencia (López Meri, 2015b). Es más, parte de la actividad de comunicación política en redes sociales está basada en la información de los medios convencionales (Marcos-García, Alonso-Muñoz y López Meri, 2021). 
En este nuevo contexto, las redes sociales se han convertido, también para los medios convencionales, en un nuevo canal a través del cual informar de manera más inmediata, transversal, y con un mayor alcance que antes (Noguera, 2010), al mismo tiempo que en una fuente informativa de gran riqueza (Suárez y Cruz, 2016; Justel-Vázquez et al., 2018). En España, según el Reuters Institute Digital News Report 2019, un 47\% de la población se informa a través de Facebook y un $16 \%$ a través de Twitter, mientras que un $52 \%$ afirma compartir noticias a través de medios sociales. Esto explica la importante presencia de los medios en estas nuevas plataformas sociales, donde, a pesar del cambio de paradigma, rápidamente han recuperado su papel como informadores de referencia y principales influenciadores de la opinión pública (McElroy, 2013).

De hecho, estudios como los de Brian Houston et al. (2020) o Hermida y Mellado (2020) han puesto el foco en el rol de Twitter (entre otras redes sociales) como fuente de información periodística, mientras Song, Gil de Zúñiga y Boomgarden (2020) han abordado cómo la forma de consumir información política a través de redes sociales puede afectar al cinismo político, demostrando lo relevante que es contribuir al estudio de estas redes como fuente de información política. También el monográfico de la revista Profesional de la Información editado por CaseroRipollés (2018) sobre información política y redes sociales ilustra la relevancia de esta materia.

\section{El uso de Twitter por los principales medios informativos españoles en períodos pre y postelectoral}

La novedad del presente estudio radica no solo en seguir ampliando el todavía incompleto cuerpo de trabajos existentes sobre el uso de Twitter como herramienta de comunicación política por parte de los medios de masas, sino también en la utilización de técnicas de recolección y análisis automatizadas que puedan seguir siendo explotadas en futuros trabajos. Así, el objetivo principal es analizar la manera en que los principales medios informativos españoles, impresos y digitales $^{1}$ - los más activos por orden de lectores-, utilizan Twitter para transmitir información política en el contexto de unas Elecciones Generales. Las elecciones que se analizan son las celebradas el 10 de noviembre de 2019, repetición de los comicios de abril del mismo año, tras los que no se formó gobierno.

La investigación amplía el estudio exploratorio realizado anteriormente por BlancoHerrero et al. (2020), centrado exclusivamente en la campaña electoral, al tiempo que complementa trabajos como los de González-Molina y Ramos del Cano (2014),

\footnotetext{
1 A lo largo de 2020 numerosos medios digitales -como El Confidencial o los del grupo Vocento- o las versiones digitales de los medios impresos - como El País o El Mundo- han incorporado diferentes modalidades de suscripción o muros de pago, lo que invita a profundizar precisamente en estos dos tipos de medios, dejando fuera del análisis a medios radiofónicos o televisivos, con un modelo de negocio y un aprovechamiento de las redes diferentes.
} 
Requejo Alemán y Herrera Damas (2011) o García de Torres et al. (2011). Dada la importancia del componente político y mediático de Twitter (D’Heer y Verdegem, 2014), el foco se coloca sobre la actividad de los principales medios de comunicación de masas en esta red social en el contexto de las citadas elecciones. Además, el trabajo distingue entre medios nativos digitales y medios impresos - aun cuando estos también cuentan con versión digital-; de esta manera, se aspira a actualizar y ampliar las observaciones realizadas por Fenoll, Cárcamo-Ulloa y Sáez-Trumper (2018), en su estudio sobre los usos de Twitter por parte de medios españoles durante periodos electorales. Para ello, planteamos:

PI1. ¿Qué diferencias hay en la cobertura política que realizan los principales medios impresos y digitales españoles en Twitter alrededor de las Elecciones Generales del 10N?

Se ha demostrado que las teorías clásicas sobre los efectos de los medios, como la agenda-setting de McCombs y Shawn (1972), también son válidas en redes sociales como Twitter (Lee y Xu, 2018; Conway, Kenski y Wang, 2015; Skogerbø y Krumsvik, 2015). Por este motivo, la presencia de un partido político en la cobertura que un medio de comunicación realiza en su cuenta de Twitter puede tener consecuencias en su desempeño electoral. Para profundizar en esta cuestión, planteamos:

PI2. ¿Qué presencia tienen los principales partidos en la cobertura que realizan los principales medios impresos y digitales españoles en Twitter alrededor de las Elecciones Generales del 10N?

Por otra parte, como ya se ha señalado, la atención prestada a la comunicación política en el marco de una campaña electoral ha sido abundante (Groshek y Al-Rawi, 2013; López García, 2016; Quevedo Redondo, Portalés-Oliva y Berrocal Gonzalo, 2016; Blanco-Herrero et al., 2020). Sin embargo, los períodos postelectorales no han recibido tanta atención, y menos todavía el análisis comparativo entre la cobertura informativa realizada antes y después de la propia jornada electoral. Una de las pocas investigaciones que sí se centra en este tipo de comparación es la desarrollada por Fenoll, Cárcamo-Ulloa y Sáez-Trumper (2018). Siguiendo la línea de estos autores, planteamos:

PI3. ¿Qué diferencias hay en la cobertura política realizada por los principales medios impresos y digitales españoles en Twitter antes y después de las Elecciones Generales del 10N?

\section{Metodología}

Se combinan técnicas cuantitativas con procesos automatizados de descarga y análisis de datos, de manera que se pudieran estudiar las publicaciones de las cuentas 
de Twitter de los cinco principales medios informativos impresos y digitales, de ámbito nacional y con mayor audiencia en España. El proceso de trabajo consistió, primero, en la selección de los medios y en la descarga de los contenidos; seguidamente, se identificó el contenido político a través de la presencia de menciones a partidos o personajes políticos - para evitar sesgos en la decisión de qué se considera política, y dado el componente exploratorio del trabajo, se usaron estas menciones como guía para considerar si un mensaje tenía índole política o no, asumiendo que en torno a unas elecciones el debate está estructurado alrededor de figuras y partidos-; por último, se procedió al análisis estadístico.

Utilizando información del Estudio General de Medios, OJD y ComScore, se seleccionaron los cinco medios impresos y cinco digitales más consumidos en el momento del análisis. Se utilizaron únicamente medios generalistas de alcance nacional, pues se buscaba que todos tuvieran el mismo ámbito y tipo de información. Para el estudio se optó por elegir la cuenta generalista de cada medio, por tener una dimensión equivalente y comparable, y por ser las que mayor número de seguidores acumulan. Las cuentas seleccionadas fueron: @el_pais (El País), @elmundoes (El Mundo), @abc_es (ABC), @larazon_es (La Razón),@20m (20 Minutos), @ elespanolcom (El Español); @elconfidencial (El Confidencial), @okdiario (okdiario), @eldiarioes (eldiario.es) y@publico_es (Público.es).

Tras la selección de cuentas se procedió a la descarga de todos los tuits publicados o retuiteados en ellas entre el 28 de octubre y el 24 de noviembre de 2019, es decir, dos semanas antes y después de la jornada electoral del 10 de noviembre de 2019. Para comparar la cobertura realizada antes y después de las elecciones se dividió la muestra en dos: entre el 28 de octubre y el 9 de noviembre, en las que se incluye la precampaña y la campaña -que en esta ocasión solamente duró una semana por tratarse de una repetición electoral-; y entre el 11 y el 24 de noviembre, en las que se evalúan los resultados y se comienza a plantear la formación del nuevo gobierno. El 10 de noviembre, día de las elecciones, se dejó fuera del análisis, ya que podría representar un problema de validez interna por no corresponderse la cobertura con la realizada ni antes ni después. Cabe destacar también que la submuestra anterior a las elecciones es de 13 días, mientras que la posterior es de 14; no obstante, esto no influía en el análisis comparativo, ya que este no se realizó con los volúmenes totales de publicaciones, sino usando medias y porcentajes, algo que no se ve afectado por los tamaños de muestra.

Para la descarga se utilizó el entorno de desarrollo integrado PyCharm ${ }^{\mathrm{TM}}$ mediante scripts personalizados en Python, haciendo uso de las librerías Tweepy y NLTK, y siguiendo la estrategia señalada previamente por Arcila et al. (2017). Específicamente, se aprovechó la Application Programming Interface (API) facilitada por Twitter para desarrolladores, a través de la función REST, que permite descargar y filtrar los tuits publicados en los últimos siete días por las cuentas seleccionadas o filtrando por hashtags o palabras clave, con un máximo de 3200 mensajes en cada 
descarga con autenticación Oauth; por lo que se tuvo que repetir esta acción en distintos momentos durante esas semanas para recopilar la muestra final.

La descarga de los mensajes de Twitter se realizó en formato JSON y se almacenó en formato csv, utilizando en el análisis los siguientes campos del track del tuit: la fecha de publicación (tweet.created_at), el número de retuits (tweet._ json['retweet_count']), el número de favoritos (tweet.json['favorite_count']) y el texto del tuit (tweet.full_text), que es el que aporta el contenido, y de donde se extrajo la información relacionada con cada uno de los partidos políticos. Así, en este campo, se estableció un sistema de codificación basado en la presencia de menciones políticas y referencias a los partidos políticos españoles y a sus principales líderes a través de palabras clave o menciones. Los términos de búsqueda para cada partido se pueden consultar en la tabla 1.

Tabla 1. Términos de búsqueda empleados para cada partido político

\begin{tabular}{|c|c|}
\hline Partido político & Términos de búsqueda \\
\hline $\begin{array}{l}\text { PSOE (Partido Socialista } \\
\text { Obrero Español) }\end{array}$ & $\begin{array}{l}\text { APSOE; Asanchezcastejon; AAdrilastra; Amjmonteroc; PSOE; Pedro } \\
\text { Sánchez (Sánchez); Adriana Lastra (Lastra); Ma (María) Jesús Montero; } \\
\text { Carmen Calvo }\end{array}$ \\
\hline PP (Partido Popular) & $\begin{array}{l}\text { Apopulares; APabloCasado_; danapastorjulian; ACayetanaAT; वTeo- } \\
\text { GarciaEgea; PP; Pablo Casado (Casado); Ana Pastor (Pastor); Álvarez } \\
\text { de Toledo; Teodoro García Egea }\end{array}$ \\
\hline Ciudadanos & $\begin{array}{l}\text { ACiudadanosCs; ACs_Madrid; AAlbert_Rivera; AlnesArrimadas; a } \\
\text { begonavillacis; Albert Rivera (Rivera); Inés Arrimadas (Arrimadas); } \\
\text { Ciudadanos; Cs; C’s }\end{array}$ \\
\hline UP (Unidas Podemos) & $\begin{array}{l}\text { dahorapodemos; APablo_Iglesias_; dilrene_Montero_; dagarzon; } \\
\text { Pablo Iglesias (Iglesias); Irene Montero (Montero); Alberto Garzón } \\
\text { (Garzón) }\end{array}$ \\
\hline Vox & $\begin{array}{l}\text { Avox_es; ASanti_ABASCAL; AmonasterioR; Q aivanedlm; Vox; Santiago } \\
\text { Abascal (Abascal); Espinosa de los Monteros; Rocío Monasterio (Mo- } \\
\text { nasterio) }\end{array}$ \\
\hline Más País & AMasPais_es; Qierrejon; Iñigo Errejón (Errejón) \\
\hline $\begin{array}{l}\text { ERC (Esquerra Republicana } \\
\text { de Catalunya) }\end{array}$ & AEsquerra_ERC; Agabrielrufian; Gabriel Rufián (Rufián); ERC \\
\hline JXCAT (Junts per Catalunya) & AJxCat; QQuimTorraiPla; Borràs; Torra \\
\hline $\begin{array}{l}\text { PNV (Partido Nacionalista } \\
\text { Vasco) }\end{array}$ & deajpnv; daitor_esteban; PNV, Aitor Esteban \\
\hline EH Bildu (Euskal Herria Bildu) & Aehbildu; AArnaldoOtegi; Arnaldo Otegi (Otegi), Bildu \\
\hline
\end{tabular}

Fuente: elaboración propia. 
Con estos términos, se identificaron los tuits que tenían contenido político y los partidos que eran mencionados en cada uno de ellos. Esta codificación se realizó manualmente con el apoyo de filtrados realizados en R Studio y clasificación de textos con recuento en tablas dinámicas. Esta labor requirió de un estudio previo del contexto de los mensajes, puesto que tanto el lenguaje utilizado como los términos con los que se designa a políticos o partidos no siempre son detectables de manera automática.

Tras añadir las etiquetas, el conjunto de datos se incorporó a la herramienta NVIVO para realizar visualizaciones exploratorias de los datos y estructurar la codificación. Por último, una vez que todos los datos fueron filtrados y etiquetados, y tras extraer la información descriptiva relevante para el estudio, se utilizó el programa SPSS (versión 26) para el análisis estadístico. En primer lugar, se recodificaron las variables que lo requerían, y se generaron nuevas variables para su operacionalización, unas categóricas, con el tipo de medio o los partidos políticos mencionados, y otras dummy, para analizar la presencia o no de contenido político en los tuits, o la mención o no de cada partido político. Una vez hecha la recodificación, se usaron las pruebas $t$ de Student para muestras independientes y ANOVA de un factor para comparar las publicaciones y las interacciones RTs (retuits) y FAVs (favoritos o me gusta) - recibidas.

\section{Resultados}

\subsection{La información política en las cuentas de Twitter de los principales medios impresos y digitales españoles en período electoral}

Como se puede apreciar en la tabla 2 , que muestra de manera desagregada el volumen total de tuits analizados, La Razón es el medio más activo de la muestra, con 6596 publicaciones (un $14.93 \%$ de todas las publicaciones del periodo). Le siguen El País y el resto de medio impresos, todos ellos por encima del medio digital más activo, eldiario.es. Así, el $60.13 \%$ del total de publicaciones fueron producidas por las cuentas de medios impresos, frente al $39.87 \%$ de medios digitales. No obstante, esta situación se invierte al centrarnos en la información política, a la que los medios digitales prestan mayor atención. En este sentido, la prueba $t$ demostró que las diferencias entre ambos tipos de medios son estadísticamente significativas $[\mathrm{t}(32144.915)=29.576 ; \mathrm{p}<.01 ; \mathrm{d}=0.29], \mathrm{y}$ que son, efectivamente, los medios digitales quienes más atención prestan a la información política; en un $26.92 \%$ de sus publicaciones hay menciones políticas $(\mathrm{DE}=0.44)$, por encima de los medios impresos, que solo dedican un $15.09 \%$ de sus tuits a esta materia $(\mathrm{DE}=0.36)$.

Además, eldiario.es, con 1522 tuits, fue el medio que mayor cantidad de publicaciones de contenido político realizó en este periodo - un 17.39\%-. Es más, de todas las publicaciones realizadas por esta cuenta en el periodo analizado, un 
$37.78 \%$ contuvieron contenido político, lo que refleja la clara apuesta del medio por este tipo de información. Todos los medios digitales muestran una atención proporcionalmente mayor a los contenidos políticos que los medios impresos, y a pesar de su menor volumen total de publicaciones, un $54.18 \%$ de los tuits sobre política fueron producidos por las cuentas de medios digitales, frente al $45.82 \%$ producido por las de medios impresos.

En lo referente a la interacción de los usuarios con la información política, reflejada a través de los RTs y FAVs, se observa que El País es el medio que lidera ambas categorías, a pesar de ser solo el cuarto en volumen de tuits políticos. Aunque serán necesarios análisis más detallados al respecto, esto puede deberse al número de seguidores de la cuenta, muy superior al resto - en el momento del estudio superaba los 7 millones de seguidores-, lo que también hace que El Mundo, con un volumen de tuits políticos más reducido —un 7.49\% del total-, esté cerca de eldiario.es, el líder en publicaciones de este tipo, debido quizá a su mayor número de seguidores - más de 3 millones, mientras que eldiario.es rondaba el millón-. Comparando el número de interacciones que generan las publicaciones de los dos tipos de medios analizados, encontramos que los tuits de los medios digitales tienen significativamente más $\mathrm{RTs}(\mathrm{M}=30.69$; $\mathrm{DE}=160.36)$, que los de prensa $(\mathrm{M}=26.37$; $\mathrm{DE}=155.8),[\mathrm{t}(36964.875)=2.805 ; \mathrm{p}<.01 ; \mathrm{d}=0.03]$. Por su parte, no se observaron diferencias significativas en el número de FAVs.

A nivel global, también se encontraron diferencias estadísticamente significativas entre los tuits de contenido político y el resto de publicaciones. Así, la prueba $t$ concluyó que los mensajes de tipo político recibieron más RTs $(\mathrm{M}=44.20 ; \mathrm{DE}=188.21)$ y más FAVs $(\mathrm{M}=67.70 ; \mathrm{DE}=324.75)$, que el resto de tuits, con una cantidad inferior tanto de RTs $(\mathrm{M}=24.11 ; \mathrm{DE}=148.87)$, [ $\mathrm{t}(11597.872)=-9.293 ; \mathrm{p}<.01 ; \mathrm{d}=0.12]$, como de FAVs $(\mathrm{M}=34.18 ; \mathrm{DE}=229.97)$, [t(11014.26) $=-8.938 ; \mathrm{p}<.01 ; \mathrm{d}=0.12]$. 
Tabla 2. Publicaciones de cada cuenta en el conjunto del periodo.

\begin{tabular}{|c|c|c|c|c|c|c|c|c|c|c|}
\hline & $\begin{array}{l}\frac{\pi}{2} \\
\stackrel{0}{\omega} \\
J\end{array}$ & $\begin{array}{l}\frac{n}{5} \\
\frac{5}{3} \\
\frac{\pi}{0} \\
\vdash\end{array}$ & 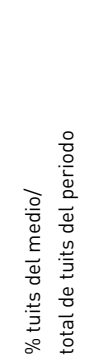 & 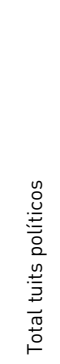 & 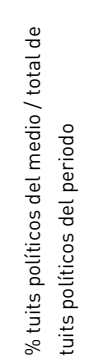 & 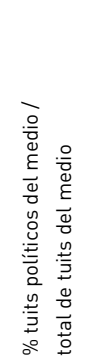 & 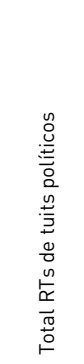 & 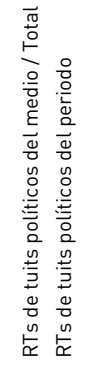 & 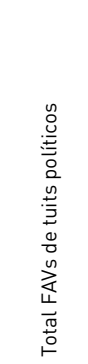 & 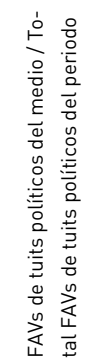 \\
\hline \multirow{5}{*}{ 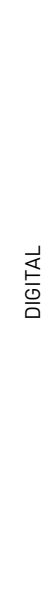 } & $\begin{array}{l}\text { elconfiden- } \\
\text { cial }\end{array}$ & 2848 & $6.44 \%$ & 667 & $7.62 \%$ & $23.42 \%$ & 32635 & $2.63 \%$ & 46536 & $2.59 \%$ \\
\hline & eldiarioes & 4029 & $9.12 \%$ & 1522 & $17.39 \%$ & $37.78 \%$ & 209375 & $16.87 \%$ & 318636 & $17.72 \%$ \\
\hline & $\begin{array}{l}\text { elespanol- } \\
\text { com }\end{array}$ & 2882 & $6.52 \%$ & 740 & $8.45 \%$ & $25.68 \%$ & 22407 & $1.81 \%$ & 34842 & $1.94 \%$ \\
\hline & okdiario & 3990 & $9.03 \%$ & 854 & $9.76 \%$ & $21.40 \%$ & 129578 & $10.44 \%$ & 185981 & $10.34 \%$ \\
\hline & publico_es & 3869 & $8.76 \%$ & 960 & $10.97 \%$ & $24.81 \%$ & 146655 & $11.81 \%$ & 149119 & $8.29 \%$ \\
\hline \multicolumn{2}{|c|}{ Total digital } & 17618 & $39.87 \%$ & 4743 & $54.18 \%$ & $26.92 \%$ & 540650 & $43.55 \%$ & 735114 & $40.88 \%$ \\
\hline \multirow{5}{*}{ 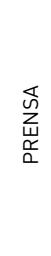 } & $20 m$ & 4918 & $11.13 \%$ & 534 & $6.10 \%$ & $10.86 \%$ & 37623 & $3.03 \%$ & 66769 & $3.71 \%$ \\
\hline & $a b c \_e s$ & 5159 & $11.67 \%$ & 768 & $8.77 \%$ & $14.89 \%$ & 116023 & $9.35 \%$ & 166265 & $9.25 \%$ \\
\hline & elmundoes & 4060 & $9.19 \%$ & 656 & $7.49 \%$ & $16.16 \%$ & 181567 & $14.63 \%$ & 256145 & $14.24 \%$ \\
\hline & el_pais & 5840 & $13.22 \%$ & 903 & $10.32 \%$ & $15.46 \%$ & 311925 & $25.13 \%$ & 499753 & $27.79 \%$ \\
\hline & larazon_es & 6596 & $14.93 \%$ & 1150 & $13.14 \%$ & $17.43 \%$ & 53525 & $4.31 \%$ & 74345 & $4.13 \%$ \\
\hline \multicolumn{2}{|c|}{ Total prensa } & 26573 & $60.13 \%$ & 4011 & $45.82 \%$ & $15.09 \%$ & 700663 & $56.45 \%$ & 1063277 & $59.12 \%$ \\
\hline \multicolumn{2}{|c|}{ Total general } & 44191 & $100.00 \%$ & 8754 & $100.00 \%$ & $19.81 \%$ & 1241313 & $100.00 \%$ & 1798391 & $100.00 \%$ \\
\hline
\end{tabular}

Fuente: elaboración propia. 


\subsection{La presencia de los principales partidos en la información política publicada por las cuentas de Twitter de los principales medios impresos y digitales españoles}

La tabla 3 muestra el reparto de menciones a los diferentes partidos políticos; como se puede observar, los porcentajes suman más del 100\%, pues hay tuits que mencionan a más de un partido. Tal y como muestra la tabla, el PSOE es el partido más mencionado a lo largo del periodo, con presencia en un $41.94 \%$ de los tuits de contenido políticos. Le sigue VOX, con aparición en un $23.51 \%$ de la muestra. Cabe destacar que existen algunas diferencias entre los distintos medios en su reparto de atención; por ejemplo, okdiario menciona al PSOE en un 56.44\% de sus tuits de contenido político, siendo el medio que mayor atención presta, de manera proporcional, a un partido.

Tabla 3. Presencia de partidos políticos en el conjunto del periodo.

\begin{tabular}{|c|c|c|c|c|c|c|c|c|c|c|c|c|c|c|}
\hline & 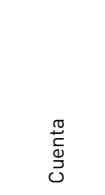 & 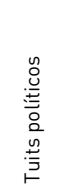 & $\begin{array}{l}\text { 山् } \\
\text { 옴 }\end{array}$ & 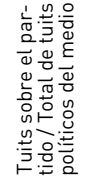 & $\frac{0}{2}$ & 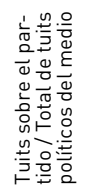 & 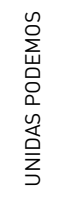 & 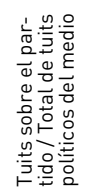 & $\stackrel{×}{\supset}$ & 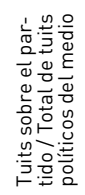 & $\begin{array}{l}\text { O } \\
\text { 充 } \\
\text { 充 } \\
\text { 号 }\end{array}$ & 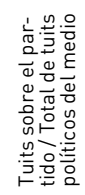 & $\begin{array}{l}\text { n } \\
\text { Oิ } \\
\text { E } \\
0\end{array}$ & 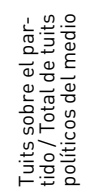 \\
\hline \multirow{5}{*}{$\frac{\frac{1}{E}}{\frac{0}{0}}$} & $\begin{array}{l}\text { elconfi- } \\
\text { dencial }\end{array}$ & 667 & 277 & $41.53 \%$ & 100 & $14.99 \%$ & 78 & $11.69 \%$ & 206 & $30.88 \%$ & 123 & $18.44 \%$ & 74 & $11.09 \%$ \\
\hline & eldiarioes & 1522 & 597 & $39.22 \%$ & 374 & $24.57 \%$ & 263 & $17.28 \%$ & 399 & $26.22 \%$ & 267 & $17.54 \%$ & 160 & $10.51 \%$ \\
\hline & $\begin{array}{l}\text { elespa- } \\
\text { nolcom }\end{array}$ & 740 & 312 & $42.16 \%$ & 150 & $20.27 \%$ & 88 & $11.89 \%$ & 158 & $21.35 \%$ & 130 & $17.57 \%$ & 115 & $15.54 \%$ \\
\hline & okdiario & 854 & 482 & $56.44 \%$ & 127 & $14.87 \%$ & 116 & $13.58 \%$ & 128 & $14.99 \%$ & 92 & $10.77 \%$ & 122 & $14.29 \%$ \\
\hline & $\begin{array}{l}\text { publi- } \\
\text { co_es }\end{array}$ & 960 & 390 & $40.63 \%$ & 161 & $16.77 \%$ & 168 & $17.50 \%$ & 282 & $29.38 \%$ & 150 & $15.63 \%$ & 74 & $7.71 \%$ \\
\hline \multicolumn{2}{|c|}{ Total digital } & 4743 & 2058 & $43.39 \%$ & 912 & $19.23 \%$ & 713 & $15.03 \%$ & 1173 & $24.73 \%$ & 762 & $16.07 \%$ & 545 & $11.49 \%$ \\
\hline \multirow{5}{*}{ 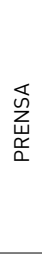 } & $20 m$ & 534 & 221 & $41.39 \%$ & 102 & $19.10 \%$ & 65 & $12.17 \%$ & 127 & $23.78 \%$ & 102 & $19.10 \%$ & 68 & $12.73 \%$ \\
\hline & $a b c \_e s$ & 768 & 355 & $46.22 \%$ & 183 & $23.83 \%$ & 98 & $12.76 \%$ & 152 & $19.79 \%$ & 139 & $18.10 \%$ & 67 & $8.72 \%$ \\
\hline & $\begin{array}{l}\text { elmun- } \\
\text { does }\end{array}$ & 656 & 312 & $47.56 \%$ & 162 & $24.70 \%$ & 108 & $16.46 \%$ & 135 & $20.58 \%$ & 100 & $15.24 \%$ & 72 & $10.98 \%$ \\
\hline & el_pais & 903 & 323 & $35.77 \%$ & 192 & $21.26 \%$ & 131 & $14.51 \%$ & 239 & $26.47 \%$ & 194 & $21.48 \%$ & 76 & $8.42 \%$ \\
\hline & $\begin{array}{l}\text { lara- } \\
\text { zon_es }\end{array}$ & 1150 & 402 & $34.96 \%$ & 275 & $23.91 \%$ & 192 & $16.70 \%$ & 232 & $20.17 \%$ & 208 & $18.09 \%$ & 110 & $9.57 \%$ \\
\hline \multicolumn{2}{|c|}{ Total prensa } & 4011 & 1613 & $40.21 \%$ & 914 & $22.79 \%$ & 594 & $14.81 \%$ & 885 & $22.06 \%$ & 743 & $18.52 \%$ & 393 & $9.80 \%$ \\
\hline \multicolumn{2}{|c|}{ Total general } & 8754 & 3671 & $41.94 \%$ & 1926 & $20.86 \%$ & 1307 & $14.93 \%$ & 2058 & $23.51 \%$ & 1505 & $17.19 \%$ & 938 & $10.72 \%$ \\
\hline
\end{tabular}

Fuente: elaboración propia. 
Por otro lado, se comparó la presencia de los cinco partidos mayoritarios - PSOE, PP, Unidas Podemos, VOX y Ciudadanos-, seleccionando solo aquellos tuits en los que un único partido de estos cinco era mencionado, y excluyendo en esta etapa del análisis aquellos contenidos en los que se aludía a más de un partido o a los partidos incluidos en la categoría de 'Otros', con la intención de centrarnos solo en los cinco partidos mencionados.

A nivel general, la prueba $t$ confirmó que estos cinco partidos tuvieron todos mayor presencia entre las cuentas de los medios digitales que en las de los impresos. Así, el PSOE recibió mayor atención en los medios digitales $(\mathrm{M}=0.12$; $\mathrm{DE}=0.32)$, que en los de prensa $(\mathrm{M}=0.06 ; \mathrm{DE}=0.24)$, $[\mathrm{t}(30203.726)=19.386 ; \mathrm{p}<.01$; $\mathrm{d}=0.21$ ]; lo mismo sucede con el PP [(Mdig=0.05; DEdig=0.22), $($ Mimp=0.03; DEimp $=0.18), \mathrm{t}(32629.47)=8.621 ; \mathrm{p}<.01 ; \mathrm{d}=0.09]$, con Unidas Podemos [(Mdig=0.04; DEdig=0.2), $($ Mimp=0.02; DEimp=0.15), $\mathrm{t}(30409,202)=10,413$; $\mathrm{p}<.01 ; \mathrm{d}=0.11]$, VOX, [(Mdig=0.07; DEdig=0.25), $($ Mimp=0.03; DEimp=0.18), $\mathrm{t}(29490.366)=15,285 ; \mathrm{p}<.01 ; \mathrm{d}=0.18]$, y Ciudadanos [(Mdig=0.04; DEdig=0.2), (Mimp=0.03; DEimp=0.17), [t(32246.452) $=8.327 ; \mathrm{p}<.01 ; \mathrm{d}=0.05]$.

Por otro lado, se midió la cantidad de retuits y favoritos que tenían los posts de cada uno de los cinco principales partidos políticos utilizando la prueba ANOVA de un factor. Así, se observaron diferencias estadísticamente significativas a nivel general en el volumen de RTs $\left[\mathrm{F}(5,8747)=9.497 ; \mathrm{p}<.01 ; \eta^{2}=0.007\right]$, así como en el volumen de FAVs $\left[F(5,8748)=12.827 ; p<.01 ; \eta^{2}=0.007\right]$. Estas diferencias comparativas entre las interacciones recogidas en los tuits que mencionan a cada uno de los partidos mayoritarios pueden visualizarse en la figura 1 .

Figura 1. Promedio general de RTs y FAVs de los tuits con menciones a cada partido mayoritario

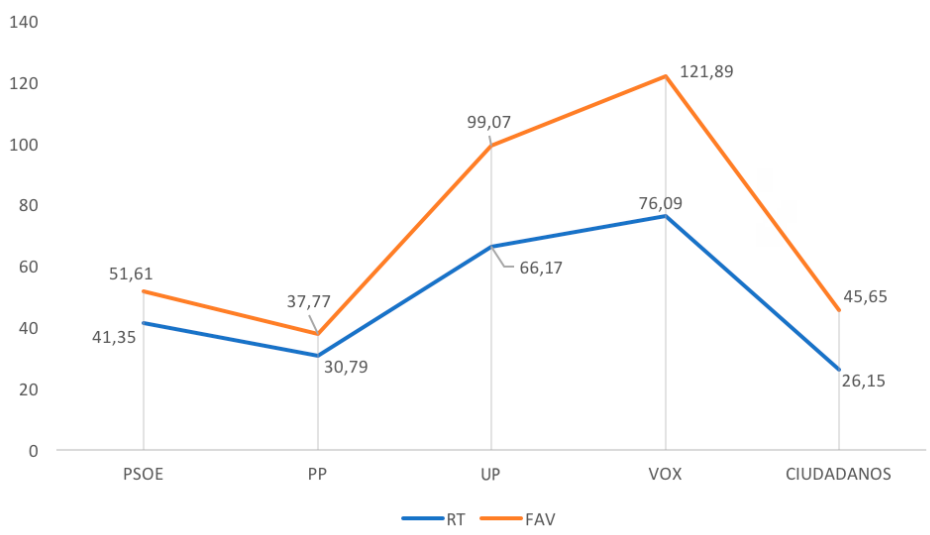

Fuente: elaboración propia. 
En relación a los RTs, las comparaciones múltiples revelaron que los tuits en los que se mencionaba a VOX $(\mathrm{M}=76.09$; $\mathrm{DE}=247.01)$ tuvieron más RTs en promedio que los que mencionaban al PSOE $(\mathrm{M}=41.35 ; \mathrm{DE}=180.73),[\mathrm{t}(8747)=5.295 ; \mathrm{p}<.01$; $\mathrm{d}=0.16]$; al PP $(\mathrm{M}=30.79 ; \mathrm{DE}=111.05)$, [t(8747)=5.616; $\mathrm{p}<.01 ; \mathrm{d}=0.24]$; y a Ciudadanos $(\mathrm{M}=26.15 ; \mathrm{DE}=189.52)$, [t(8747)=6.352; $\mathrm{p}<.01 ; \mathrm{d}=0.23]$. Igualmente, los tuits con menciones a UP $(\mathrm{M}=66.17 ; \mathrm{DE}=243.56)$ tuvieron más RTs que los referidos al PP $[\mathrm{t}(8747)=3.356 ; \mathrm{p}<.05 ; \mathrm{d}=0.19]$ y a Ciudadanos $[\mathrm{t}(8747)=3.853 ; \mathrm{p}<.01 ; \mathrm{d}=0.09]$.

Respecto a los FAVs, las comparaciones múltiples manifestaron que los tuits con menciones a VOX obtuvieron en promedio un mayor número $(\mathrm{M}=121.89$; $\mathrm{DE}=401.98)$ que los que aluden al PSOE $(\mathrm{M}=51.61 ; \mathrm{DE}=246.14)$, $[\mathrm{t}(8748)=5.812$; $\mathrm{p}<.01 ; \mathrm{d}=0.21]$; al PP $(\mathrm{M}=37.77 ; \mathrm{DE}=126.55)$, [t(8748)=7.280; $\mathrm{p}<.01 ; \mathrm{d}=0.28]$; y a Ciudadanos $[(\mathrm{M}=45.65 ; \mathrm{DE}=290.96), \mathrm{t}(8748)=5.343, \mathrm{p}<.01, \mathrm{~d}=0.22]$. Por otro lado, los tuits con referencias a UP tuvieron significativamente más FAVs $(\mathrm{M}=99.07$; $\mathrm{DE}=366.02)$, que los que incluían alusiones al PP [t(8748)=3.604; $\mathrm{p}<.01 ; \mathrm{d}=0.22]$.

\subsection{Cambios en la información política en las cuentas de Twitter de los principales medios impresos y digitales españoles antes y después de la jornada electoral}

Como se aprecia en la tabla 4, el comportamiento general no cambia antes y después de las elecciones, y son los medios impresos los que más publican en ambas sub-muestras, manteniéndose también los digitales como los que mayor atención prestan a la información política. Así, en las semanas anteriores a la jornada electoral, los medios digitales dedicaron un 27.89\% (DE=0.449) de sus tuits a contenidos políticos, mientras que las cuentas de medios convencionales dedicaron solamente un $16.04 \%$ ( $\mathrm{DE}=0.37)$, lo que supone una diferencia estadísticamente significativa [t(16231.885) $=20.479 ; \mathrm{p}<.01 ; \mathrm{d}=0.29]$. Lo mismo ocurre en el período postelectoral, cuando los medios digitales continúan publicando más tuits políticos, un 25.96\% (DE=0.438) de sus posts, que los medios de prensa, un $14.21 \%$ $(\mathrm{DE}=0.35),[\mathrm{t}(15911.606)=21.274 ; \mathrm{p}<.01 ; \mathrm{d}=0.30]$.

Sin embargo, sí se aprecian cambios en el porcentaje de tuits políticos, que disminuye significativamente, pasando de un 20.84\% (DE=0.406) antes de las elecciones a un $18.82 \%(\mathrm{DE}=0.39)$ después de las mismas $[\mathrm{t}(43883.804)=5.316 ; \mathrm{p}<.01$; $\mathrm{d}=0.05]$, demostrando la mayor presencia que tiene la información política durante la campaña, superando a la etapa de formación de gobierno. Este descenso es particularmente visible en el caso de eldiario.es que, aunque sigue siendo la cuenta que más interés muestra por la información política, pasa de un $41.50 \%$ antes de la jornada electoral a un 34.01 \% tras ella. El descenso es también abultado en La Razón, que pasa de ser el medio impreso que más interés político mostraba antes de las elecciones, con un $21.50 \%$ de sus tuits, a ser, con un $13.62 \%$, el segundo menos interesado en política, solo por delante de 20 minutos, con un $11.14 \%$. 
Tabla 4. Publicaciones de cada cuenta antes y después de las elecciones

\begin{tabular}{|c|c|c|c|c|c|c|c|c|c|c|}
\hline \multicolumn{11}{|c|}{ Período pre-electoral } \\
\hline & $\begin{array}{l}\frac{\pi}{0} \\
\stackrel{0}{0} \\
J \\
0\end{array}$ & $\begin{array}{l}\frac{n}{5} \\
\frac{.}{2} \\
\frac{\pi}{0} \\
0 \\
⺊\end{array}$ & 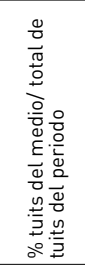 & 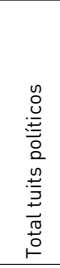 & 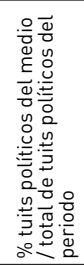 & 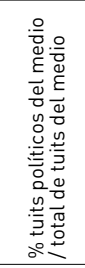 & 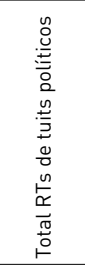 & 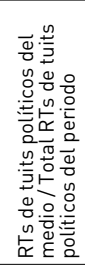 & 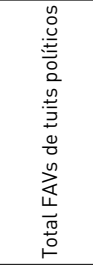 & 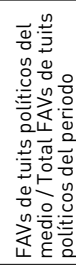 \\
\hline \multirow{5}{*}{$\frac{\frac{1}{\mathbb{E}}}{\frac{0}{\square}}$} & elconfidencial & 1404 & $6.50 \%$ & 321 & $7.13 \%$ & $22.86 \%$ & 10980 & $2.09 \%$ & 15557 & $2.05 \%$ \\
\hline & eldiarioes & 2024 & $9.37 \%$ & 840 & $18.66 \%$ & $41.50 \%$ & 96767 & $18.39 \%$ & 125496 & $16.50 \%$ \\
\hline & elespanolcom & 1449 & $6.71 \%$ & 405 & $9.00 \%$ & $27.95 \%$ & 10587 & $2.01 \%$ & 16042 & $2.11 \%$ \\
\hline & okdiario & 1919 & $8.89 \%$ & 385 & $8.55 \%$ & $20.06 \%$ & 58830 & $11.18 \%$ & 88524 & $11.64 \%$ \\
\hline & publico_es & 1948 & $9.02 \%$ & 488 & $10.84 \%$ & $25.05 \%$ & 46590 & $8.85 \%$ & 50727 & $6.67 \%$ \\
\hline \multicolumn{2}{|c|}{ Total digital } & 8744 & $40.49 \%$ & 2439 & $54.19 \%$ & $27.89 \%$ & 223754 & $42.52 \%$ & 296346 & $38.97 \%$ \\
\hline \multirow{5}{*}{ 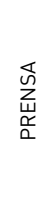 } & $20 m$ & 2431 & $11.26 \%$ & 257 & $5.71 \%$ & $10.57 \%$ & 16720 & $3.18 \%$ & 28305 & $3.72 \%$ \\
\hline & abc_es & 2442 & $11.31 \%$ & 352 & $7.82 \%$ & $14.41 \%$ & 54256 & $10.31 \%$ & 78030 & $10.26 \%$ \\
\hline & elmundoes & 1952 & $9.04 \%$ & 282 & $6.27 \%$ & $14.45 \%$ & 77046 & $14.64 \%$ & 109540 & $14.40 \%$ \\
\hline & el_pais & 2832 & $13.11 \%$ & 484 & $10.75 \%$ & $17.09 \%$ & 137886 & $26.20 \%$ & 225114 & $29.60 \%$ \\
\hline & larazon_es & 3196 & $14.80 \%$ & 687 & $15.26 \%$ & $21.50 \%$ & 16588 & $3.15 \%$ & 23154 & $3.04 \%$ \\
\hline \multicolumn{2}{|c|}{ Total prensa } & 12853 & $59.51 \%$ & 2062 & $45.81 \%$ & $16.04 \%$ & 302496 & $57.48 \%$ & 464143 & $61.03 \%$ \\
\hline \multicolumn{2}{|c|}{ Total general } & 21597 & $100 \%$ & 4501 & $100 \%$ & $20.84 \%$ & 526250 & $100 \%$ & 760489 & $100 \%$ \\
\hline \multicolumn{11}{|c|}{ Período post-electoral } \\
\hline \multirow{5}{*}{$\frac{\frac{1}{5}}{\frac{0}{0}}$} & elconfidencial & 1444 & $6.39 \%$ & 346 & $8.14 \%$ & $23.96 \%$ & 21655 & $3.03 \%$ & 30979 & $2.98 \%$ \\
\hline & eldiarioes & 2005 & $8.87 \%$ & 682 & $16.04 \%$ & $34.01 \%$ & 112608 & $15.75 \%$ & 193140 & $18.61 \%$ \\
\hline & elespanolcom & 1433 & $6.34 \%$ & 335 & $7.88 \%$ & $23.38 \%$ & 11820 & $1.65 \%$ & 18800 & $1.81 \%$ \\
\hline & okdiario & 2071 & $9.17 \%$ & 469 & $11.03 \%$ & $22.65 \%$ & 70748 & $9.89 \%$ & 97457 & $9.39 \%$ \\
\hline & publico_es & 1921 & $8.50 \%$ & 472 & $11.10 \%$ & $24.57 \%$ & 100065 & $13.99 \%$ & 98392 & $9.48 \%$ \\
\hline \multicolumn{2}{|c|}{ Total digital } & 8874 & $39.28 \%$ & 2304 & $54.17 \%$ & $25.96 \%$ & 316896 & $44.32 \%$ & 438768 & $42.27 \%$ \\
\hline \multirow{5}{*}{ 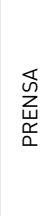 } & $20 \mathrm{~m}$ & 2487 & $11.01 \%$ & 277 & $6.51 \%$ & $11.14 \%$ & 20903 & $2.92 \%$ & 38464 & $3.71 \%$ \\
\hline & abc_es & 2717 & $12.03 \%$ & 416 & $9.78 \%$ & $15.31 \%$ & 61767 & $8.64 \%$ & 88235 & $8.50 \%$ \\
\hline & elmundoes & 2108 & $9.33 \%$ & 374 & $8.79 \%$ & $17.74 \%$ & 104521 & $14.62 \%$ & 146605 & $14.13 \%$ \\
\hline & el_pais & 3008 & $13.31 \%$ & 419 & $9.85 \%$ & $13.93 \%$ & 174039 & $24.34 \%$ & 274639 & $26.46 \%$ \\
\hline & larazon_es & 3400 & $15.05 \%$ & 463 & $10.89 \%$ & $13.62 \%$ & 36937 & $5.17 \%$ & 51191 & $4.93 \%$ \\
\hline \multicolumn{2}{|c|}{ Total prensa } & 13720 & $60.72 \%$ & 1949 & $45.83 \%$ & $14.21 \%$ & 398167 & $55.68 \%$ & 599134 & $57.73 \%$ \\
\hline \multicolumn{2}{|c|}{ Total general } & 22594 & $100 \%$ & 4253 & $100 \%$ & $18.82 \%$ & 715063 & $100 \%$ & 1037902 & $100 \%$ \\
\hline
\end{tabular}

Fuente: elaboración propia. 
También se observaron pequeñas diferencias entre el promedio de retuits y de favoritos generados por las publicaciones políticas en el período anterior a las elecciones y en el posterior. Así, tras la jornada electoral hubo más RTs $(\mathrm{M}=47.89$; $\mathrm{DE}=196.35)$ que en los previos $(\mathrm{M}=40.71 ; \mathrm{DE}=180.12)$, aunque la diferencia solo podría considerarse tendencial $[\mathrm{t}(8577.434)=-1.778 ; \mathrm{p}<.08]$. También se observaron más FAVs tras las elecciones $(\mathrm{M}=75.44$; $\mathrm{DE}=373.32)$ que antes de las mismas $(\mathrm{M}=59.16$; $\mathrm{DE}=270.77)$, y en este caso la prueba $t$ sí arrojó diferencias estadísticamente significativas $[\mathrm{t}(7726.038)=-2.324 ; \mathrm{p}<.05 ; \mathrm{d}=0.05]$.

Los cambios más reseñables, no obstante, se producen en el interés que despierta cada partido, que depende de sus expectativas y de los datos de encuestas en el periodo pre-electoral, y del resultado electoral y las posibilidades de formar gobierno en el postelectoral. Así, en la tabla 5 se puede observar que, si bien el PSOE había sido el partido que mayor interés despertaba durante las semanas previas a las elecciones, presente en un $35.64 \%$ de las publicaciones, en las semanas que suceden a la jornada electoral ese porcentaje asciende hasta el $47.54 \%$, pues se trataba del ganador de las elecciones y del único partido con opciones de formar gobierno. También fue muy importante el crecimiento de UP, que pasó de aparecer en un $8.60 \%$ de los tuits antes del $10 \mathrm{~N}$ a hacerlo en el $21.63 \%$ tras esa fecha; esto puede explicarse por el rápido acuerdo firmado con el PSOE para intentar formar un gobierno de coalición. De cara a lograr los apoyos suficientes, las negociaciones incluyeron a partidos de menor tamaño, que formaban parte de la categoría 'Otros'; con esto, también crecieron las menciones de este grupo de partidos, pasando de un $7.73 \%$ a un $13.87 \%$. Estos datos no detallan la atención hacia cada uno de los partidos minoritarios incluidos en el análisis - Más País, ERC, JXCAT, PNV y Bildu-; de la misma forma, partidos con menor representación, como el Partido Regionalista de Cantabria, Teruel Existe o la CUP, no fueron incluidos en el análisis y no se puede evaluar si despertaron una atención creciente o decreciente tras las elecciones. Por su parte, PP y VOX, a pesar de su aumento en escaños, perdieron presencia tras las elecciones, momento en el que la información se centró en la mencionada formación de gobierno. Por último, puede resultar llamativo que Ciudadanos, sin un rol determinante en dicha formación de gobierno, ganara presencia tras las elecciones, pasando a estar presente en un $17.89 \%$ de tuits políticos, frente al $16.53 \%$ de los tuits sobre política antes de las elecciones. Esto puede deberse precisamente a su gran caída en votos y escaños y a las consecuencias que esto tuvo. 
Tabla 5. Presencia de partidos políticos en los tuits publicados por los principales medios antes y después de las elecciones

\begin{tabular}{|c|c|c|c|c|c|c|c|c|c|c|c|c|c|c|}
\hline \multicolumn{15}{|c|}{ Período pre-electoral } \\
\hline & $\begin{array}{l}\underset{\pi}{\sim} \\
\stackrel{0}{J} \\
0\end{array}$ & 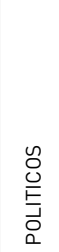 & $\begin{array}{l}\text { 山् } \\
\text { ณ }\end{array}$ & 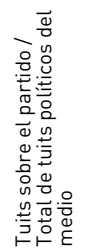 & 음 & 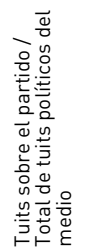 & 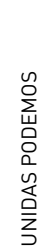 & 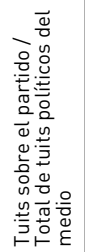 & $\stackrel{\times}{\rho}$ & 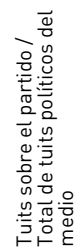 & 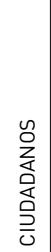 & 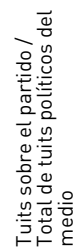 & 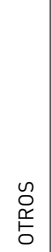 & 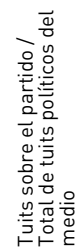 \\
\hline \multirow{5}{*}{$\frac{\frac{d}{5}}{\frac{\Xi}{0}}$} & $\begin{array}{l}\text { elconfiden- } \\
\text { cial }\end{array}$ & 321 & 113 & $35.20 \%$ & 54 & $16.82 \%$ & 18 & $5.61 \%$ & 101 & $31.46 \%$ & 60 & $18.69 \%$ & 28 & $8.72 \%$ \\
\hline & eldiarioes & 840 & 279 & $33.21 \%$ & 222 & $26.43 \%$ & 80 & $9.52 \%$ & 257 & $30.60 \%$ & 131 & $15.60 \%$ & 62 & $7.38 \%$ \\
\hline & $\begin{array}{l}\text { elespanol- } \\
\text { com }\end{array}$ & 405 & 145 & $35.80 \%$ & 73 & $18.02 \%$ & 22 & $5.43 \%$ & 95 & $23.46 \%$ & 71 & $17.53 \%$ & 51 & $12.59 \%$ \\
\hline & okdiario & 385 & 201 & $52.21 \%$ & 60 & $15.58 \%$ & 21 & $5.45 \%$ & 73 & $18.96 \%$ & 52 & $13.51 \%$ & 30 & $7.79 \%$ \\
\hline & publico_es & 488 & 189 & $38.73 \%$ & 92 & $18.85 \%$ & 42 & $8.61 \%$ & 152 & $31.15 \%$ & 55 & $11.27 \%$ & 35 & $7.17 \%$ \\
\hline \multicolumn{2}{|c|}{ Total digital } & 2439 & 927 & $38.01 \%$ & 501 & $20.54 \%$ & 183 & $7.50 \%$ & 678 & $27.80 \%$ & 369 & $15.13 \%$ & 206 & $8.45 \%$ \\
\hline \multirow{5}{*}{ 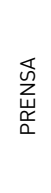 } & $20 \mathrm{~m}$ & 257 & 94 & $36.58 \%$ & 54 & $21.01 \%$ & 24 & $9.34 \%$ & 66 & $25.68 \%$ & 52 & $20.23 \%$ & 30 & $11.67 \%$ \\
\hline & abc_es & 352 & 159 & $45.17 \%$ & 99 & $28.13 \%$ & 27 & $7.67 \%$ & 81 & $23.01 \%$ & 65 & $18.47 \%$ & 23 & $6.53 \%$ \\
\hline & elmundoes & 282 & 120 & $42.55 \%$ & 76 & $26.95 \%$ & 28 & $9.93 \%$ & 68 & $24.11 \%$ & 48 & $17.02 \%$ & 25 & $8.87 \%$ \\
\hline & el_pais & 484 & 145 & $29.96 \%$ & 96 & $19.83 \%$ & 48 & $9.92 \%$ & 139 & $28.72 \%$ & 95 & $19.63 \%$ & 25 & $5.17 \%$ \\
\hline & larazon_es & 687 & 204 & $29.69 \%$ & 174 & $25.33 \%$ & 77 & $11.21 \%$ & 149 & $21.69 \%$ & 115 & $16.74 \%$ & 39 & $5.68 \%$ \\
\hline \multicolumn{2}{|c|}{ Total prensa } & 2062 & 722 & $35.01 \%$ & 499 & $24.20 \%$ & 204 & $9.89 \%$ & 503 & $24.39 \%$ & 375 & $18.19 \%$ & 142 & $6.89 \%$ \\
\hline \multicolumn{2}{|c|}{ Total general } & 4501 & 1649 & $36.64 \%$ & 1000 & $22.22 \%$ & 387 & $8.60 \%$ & 1181 & $26.24 \%$ & 744 & $16.53 \%$ & 348 & $7.73 \%$ \\
\hline \multicolumn{15}{|c|}{ Período post-electoral } \\
\hline \multirow{5}{*}{ 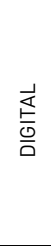 } & $\begin{array}{l}\text { elconfiden- } \\
\text { cial }\end{array}$ & 346 & 164 & $47.40 \%$ & 46 & $13.29 \%$ & 60 & $17.34 \%$ & 105 & $30.35 \%$ & 63 & $18.21 \%$ & 46 & $13.29 \%$ \\
\hline & eldiarioes & 682 & 318 & $46.63 \%$ & 152 & $22.29 \%$ & 183 & $26.83 \%$ & 142 & $20.82 \%$ & 136 & $19.94 \%$ & 98 & $14.37 \%$ \\
\hline & $\begin{array}{l}\text { elespanol- } \\
\text { com }\end{array}$ & 335 & 167 & $49.85 \%$ & 77 & $22.99 \%$ & 66 & $19.70 \%$ & 63 & $18.81 \%$ & 59 & $17.61 \%$ & 64 & $19.10 \%$ \\
\hline & okdiario & 469 & 281 & $59.91 \%$ & 67 & $14.29 \%$ & 95 & $20.26 \%$ & 55 & $11.73 \%$ & 40 & $8.53 \%$ & 92 & $19.62 \%$ \\
\hline & publico_es & 472 & 201 & $42.58 \%$ & 69 & $14.62 \%$ & 126 & $26.69 \%$ & 130 & $27.54 \%$ & 95 & $20.13 \%$ & 39 & $8.26 \%$ \\
\hline \multicolumn{2}{|c|}{ Total digital } & 2304 & 1131 & $49.09 \%$ & 411 & $17.84 \%$ & 530 & $23.00 \%$ & 495 & $21.48 \%$ & 393 & $17.06 \%$ & 339 & $14.71 \%$ \\
\hline \multirow{5}{*}{ 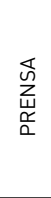 } & $20 \mathrm{~m}$ & 277 & 127 & $45.85 \%$ & 48 & $17.33 \%$ & 41 & $14.80 \%$ & 61 & $22.02 \%$ & 50 & $18.05 \%$ & 38 & $13.72 \%$ \\
\hline & abc_es & 416 & 196 & $47.12 \%$ & 84 & $20.19 \%$ & 71 & $17.07 \%$ & 71 & $17.07 \%$ & 74 & $17.79 \%$ & 44 & $10.58 \%$ \\
\hline & elmundoes & 374 & 192 & $51.34 \%$ & 86 & $22.99 \%$ & 80 & $21.39 \%$ & 67 & $17.91 \%$ & 52 & $13.90 \%$ & 47 & $12.57 \%$ \\
\hline & el_pais & 419 & 178 & $42.48 \%$ & 96 & $22.91 \%$ & 83 & $19.81 \%$ & 100 & $23.87 \%$ & 99 & $23.63 \%$ & 51 & $12.17 \%$ \\
\hline & larazon_es & 463 & 198 & $42.76 \%$ & 101 & $21.81 \%$ & 115 & $24.84 \%$ & 83 & $17.93 \%$ & 93 & $20.09 \%$ & 71 & $15.33 \%$ \\
\hline \multicolumn{2}{|c|}{ Total prensa } & 1949 & 891 & $45.72 \%$ & 415 & $21.29 \%$ & 390 & $20.01 \%$ & 382 & $19.60 \%$ & 368 & $18.88 \%$ & 251 & $12.88 \%$ \\
\hline \multicolumn{2}{|c|}{ Total general } & 4253 & 2022 & $47.54 \%$ & 826 & $19.42 \%$ & 920 & $21.63 \%$ & 877 & $20.62 \%$ & 761 & $17.89 \%$ & 590 & $13.87 \%$ \\
\hline
\end{tabular}

Fuente: elaboración propia. 
Centrando la comparación en los cinco principales partidos políticos, la prueba $t$ manifestó que los que finalmente formarían gobierno vieron incrementada de manera significativa su presencia tras la jornada electoral. Así, el número de mensajes relacionados con el PSOE fue inferior antes $(\mathrm{M}=0.08$; $\mathrm{DE}=0.27)$ que después de la votación $(\mathrm{M}=0.09 ; \mathrm{DE}=0.29)$, [t(44156.647)=-5.012; $\mathrm{p}<.01 ; \mathrm{d}=0.04]$. Y lo mismo sucedió con los tuits que mencionaban a UP [(Mpre $=0.02 ;$ DEpre $=0.13)$ $($ Mpos=0.04; DEpos=0.2), t $(39686.202)=-14.295 ; \mathrm{p}<.01 ; \mathrm{d}=0.12]$. Respecto al PP y VOX, ambos vieron reducida la proporción de tuits que les mencionaba tras la jornada electoral. Así, el PP pasó de aparecer en un 5\% de los tuits antes de las elecciones $(\mathrm{M}=0.05 ; \mathrm{DE}=0.21)$, a un $4 \%$ de la muestra tras los comicios $(\mathrm{M}=0.04$; $\mathrm{DE}=0.19),[\mathrm{t}(43126.811)=5.11 ; \mathrm{p}<.01 ; \mathrm{d}=0.05]$, mientras VOX experimentó una reducción muy semejante [ $(\mathrm{Mpre}=0.05$; DEpre $=0.23)$, $($ Mpos $=0.04$; DEpos $=0.19)$, $\mathrm{t}(42388.043)=7.89 ; \mathrm{p}<.01 ; \mathrm{d}=0.05]$. Ciudadanos, sin embargo, no experimentó ningún cambio estadísticamente significativo entre ambas etapas.

Para terminar, se llevó a cabo un análisis comparativo por cada uno de los cinco primeros partidos políticos para confirmar si se había producido un cambio en la media de retuits y de favoritos que recibieron las publicaciones en las que se mencionaba a cada uno de ellos antes y después de las elecciones. En este caso, la prueba $t$ arrojó diferencias estadísticamente significativas entre el período preelectoral y el postelectoral tan solo en los mensajes de Ciudadanos y de VOX, aunque en sentidos diferentes. Así, los mensajes de VOX, que se habían reducido significativamente tras la jornada electoral, también recibieron de promedio más RTs antes $(\mathrm{M}=88.12 ; \mathrm{DE}=293.12)$ que después de las elecciones $(\mathrm{M}=59.31 ; \mathrm{DE}=160.86)$, $[\mathrm{t}(1318.793)=2.356 ; \mathrm{p}<.05 ; \mathrm{d}=0.12]$; del mismo modo, también recibieron más FAVs en el período de campaña $(\mathrm{M}=146.41 ; \mathrm{DE}=477.47)$ que en el posterior a los comicios $(\mathrm{M}=87.70 ; \mathrm{DE}=259.22)$, $[\mathrm{t}(1313.145)=2.957 ; \mathrm{p}<.01 ; \mathrm{d}=0.15]$. Mientras tanto, los tuits de menciones a Ciudadanos, que no habían experimentado cambio significativo en cuanto a cantidad de publicaciones, sí que presentan un incremento en RTs entre el período pre $(\mathrm{M}=15.27 ; \mathrm{DE}=42.44)$ y postelectoral $(\mathrm{M}=35.73$; $\mathrm{DE}=256.56),[\mathrm{t}(540.538)=-1.773 ; \mathrm{p}<.01 ; \mathrm{d}=0.11]$; así como FAVs, también con un mayor número antes $(\mathrm{M}=25.71 ; \mathrm{DE}=73.06)$ que después de las elecciones $(\mathrm{M}=63.19 ; \mathrm{DE}=392.4),[\mathrm{t}(548.906)=-2.116 ; \mathrm{p}<.01 ; \mathrm{d}=0.13]$.

Estos cambios dieron lugar a una menor diferenciación entre partidos en el periodo postelectoral, como se puede apreciar en la figura 2. Así, la prueba ANOVA de un factor arrojó diferencias estadísticamente significativas entre los tuits de los cinco principales partidos en RTs $\left[F(5,4494)=14.308 ; p<.01 ; \eta^{2}=0.018\right]$ y en FAVs $\left[F(5,4495)=12.175 ; p<.01 ; \eta^{2}=0.025\right]$ de mayor tamaño durante el periodo previo al $10 \mathrm{~N}$, si bien es cierto que estas diferencias siguieron siendo significativas tras las elecciones, tanto en el promedio de RTs $\left[F(5,4247)=3.073 ; p<.01 ; \eta^{2}=0.003\right]$ como de FAVs $\left[\mathrm{F}(5,4247)=3.384 ; \mathrm{p}<.01 ; \eta^{2}=0.002\right]$. 
Detalladas por partidos y periodos, las comparaciones múltiples revelaron que, antes de la jornada electoral, los mensajes con menciones a VOX tuvieron más RTs $(\mathrm{M}=88.12 ; \mathrm{DE}=293.12)$ que los que mencionaban al PSOE $(\mathrm{M}=38.23 ; \mathrm{DE}=207.03)$, $[\mathrm{t}(4494)=4.147 ; \mathrm{p}<.01 ; \mathrm{d}=0.19] ;$ al PP $(\mathrm{M}=31.19 ; \mathrm{DE}=113.07),[\mathrm{t}(4494)=4.992 ; \mathrm{p}<.01$; $\mathrm{d}=0.26]$; y a Ciudadanos $(\mathrm{M}=15.27$; $\mathrm{DE}=42.44)$, [t(4494)=6.968; $\mathrm{p}<.01 ; \mathrm{d}=0.35]$. Mientras tanto, los tuits con menciones de Ciudadanos tuvieron en promedio menos RTs que los del PSOE [t(4494)=3.483; $<<0.01 ; \mathrm{d}=0.15]$; los del PP [t(4494)=2.966; $\mathrm{p}<.05 ; \mathrm{d}=0.19]$; $\mathrm{y}$ los de UP $(\mathrm{M}=56.41 ; \mathrm{DE}=178.23)$, [t(4494)=3.362; $\mathrm{p}<.05 ; \mathrm{d}=0.32]$. Respecto a los favoritos, antes de la jornada electoral los tuits con aparición de VOX también fueron los que más FAVs tuvieron $(\mathrm{M}=146.41$; $\mathrm{DE}=477.47)$, significativamente por encima de los que tenían menciones al PSOE $(\mathrm{M}=45.27 ; \mathrm{DE}=261.31)$, $[\mathrm{t}(4495)=5.467 ; \mathrm{p}<.01 ; \mathrm{d}=0.26] ;$ al PP $(\mathrm{M}=33.07 ; \mathrm{DE}=99.01),[\mathrm{t}(4495)=6.561 ; \mathrm{p}<.01$; $\mathrm{d}=0.33$ ]; $\mathrm{y}$ a Ciudadanos $(\mathrm{M}=25.71 ; \mathrm{DE}=73.06)$, [t(4494)=7.072; $\mathrm{p}<.01 ; \mathrm{d}=0.35]$. Los tuits con aparición de Ciudadanos, a su vez, fueron los que menos FAVs tuvieron, presentando diferencias significativas también con los que incluían referencias a UP $(\mathrm{M}=88.87 ; \mathrm{DE}=288.71)$, [t(4495)=3.180; $\mathrm{p}<.05 ; \mathrm{d}=0.30]$.

En el periodo postelectoral las diferencias estadísticamente significativas se dieron solo entre dos de los partidos mayoritarios. En base a la prueba estadística, aunque el partido que más interacciones presentaba tras las elecciones fue UP, las diferencias significativas sólo tuvieron lugar entre los tuits que aludían a VOX -el segundo en interacciones tras UP- y los que aludían al PP, siendo los primeros los que más RTs $(\mathrm{M}=59.31$; $\mathrm{DE}=160.86)$ y más FAVs $(\mathrm{M}=87.70 ; \mathrm{DE}=259.22)$ tenían, frente a los mensajes con menciones al PP, que tuvieron significantemente menos RTs $(\mathrm{M}=30.24 ; \mathrm{DE}=108.27)$, [t(4247)=3.327; $\mathrm{p}<.05 ; \mathrm{d}=0.21]$, y menos FAVs $(\mathrm{M}=44.42 ; \mathrm{DE}=157.4),[\mathrm{t}(4247)=3.202 ; \mathrm{p}<.05 ; \mathrm{d}=0.20]$. 
Figura 2. Promedio de RTs y FAVs de los tuits con menciones a los cinco partidos políticos mayoritarios antes y después de las elecciones
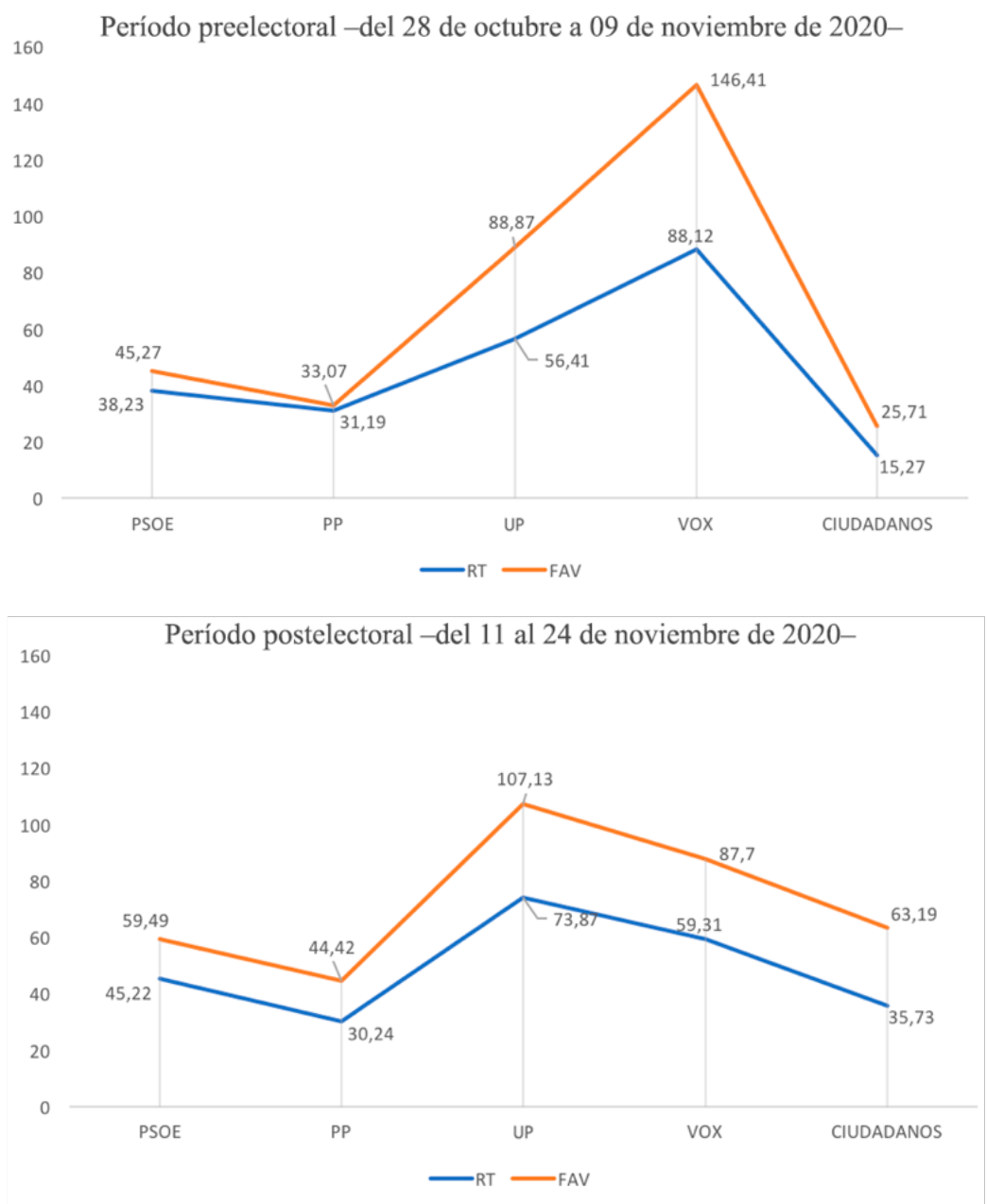

Fuente: elaboración propia. 


\section{Conclusiones y discusión}

Respondiendo a la primera pregunta de investigación, se ha observado que, aunque los medios impresos tienen una mayor actividad en sus cuentas de Twitter, son los medios digitales los que prestan una mayor atención a la información política, además de ser los que más interacción generan en términos de RTs y FAVs, algo que ya se apuntaba en el trabajo exploratorio publicado anteriormente (Blanco-Herrero et al., 2020). La diferencia en RTs y FAVs en los mensajes con contenido político puede deberse al hecho de que este tipo de información tenga un mayor grado de impacto social y, por lo tanto, genere mayor interacción que el resto de publicaciones. No obstante, convendrá seguir estudiando las diferencias en la atención que los dos tipos de medios prestan al contenido político, profundizando también en las cuestiones formales del tuit - presencia de hashtags, menciones o URLs- que habían abordado anteriormente Fenoll, Cárcamo-Ulloa y Sáez-Trumper (2018).

En lo referente a la presencia de los diferentes partidos políticos, y respondiendo a la segunda pregunta de investigación, se observa que PSOE y VOX son los partidos con mayor presencia en el conjunto de la muestra. Esto también se denotaba en los resultados del trabajo centrado en el período preelectoral (Blanco-Herrero et al., 2020), algo que ocurría de igual forma en las cuentas de los principales medios radiofónicos y televisivos. Esto no sorprende ya que, en el caso del PSOE, se trata del partido que se situaba en el gobierno en funciones, que encabezaba las encuestas, y que tras las elecciones se convertiría en el partido más votado y líder de la posterior coalición de gobierno. En el caso de VOX, se trata del partido que experimentó el mayor crecimiento en escaños, logrando un resultado histórico para una formación que había obtenido representación parlamentaria por primera vez en las anteriores elecciones, en abril del mismo año, y que además se presentaba con un discurso radical y todavía novedoso en el panorama político español (Turnbull-Dugarte, Rama y Santana, 2020). Esta radicalidad parece explicar también el nivel de interacción, pues VOX es, junto con el partido situado en el extremo opuesto, UP, quienes más RTs y FAVs generaron.

Lo anterior, que es válido para el conjunto de la muestra, debe matizarse con la respuesta a la tercera pregunta de investigación, pues hubo diferencias importantes entre el periodo anterior y el posterior a las elecciones. Así, los datos indican que hubo más tuits con contenido político en el período preelectoral que en el postelectoral, algo comprensible al constituir las campañas electorales un fenómeno de elevado interés mediático. Sin embargo, el interés por partidos se vio muy influido por el resultado obtenido en las urnas, de manera que, de los cinco mayoritarios, los dos que finalmente formaron gobierno - PSOE y UP - vieron incrementada la cantidad de tuits que les mencionaban tras las elecciones, mientras que PP y VOX, partidos que crecieron en escaños pero que no participaron en los pactos para formar gobierno, experimentaron el fenómeno contrario. Por su parte, la cantidad de 
menciones a Ciudadanos permanece estable tras las elecciones, ya que la prueba estadística no arrojó diferencias significativas en este caso.

En lo referente a las interacciones, fueron mayores en el periodo posterior a la jornada electoral, lo cual contrasta con esa menor proporción de contenidos políticos tras las elecciones, por lo que serán necesarios más análisis en esta línea para conocer la evolución de la información política en las cuentas de Twitter de los medios de comunicación antes y después de unas elecciones. Cabe añadir que, a nivel de cada partido, se encontraron diferencias significativas en el volumen de interacciones tan solo en los mensajes con menciones a dos de ellos; por un lado VOX, con un descenso significativo tanto en RTs como en FAVs; y por otro lado Ciudadanos, un caso especial ya que, a pesar de mantener una cantidad estable de tuits antes y después de las elecciones, los mensajes de este partido generaron una mayor cantidad de RTs y de FAVs en el período postelectoral. La razón de este cambio, en el caso de VOX, pudo ser su ausencia en la formación de gobierno tras una campaña en la que tuvo un rol protagonista. Por su parte, el incremento en interacciones de los tuits de Ciudadanos pudo deberse al fracaso electoral de este partido, que sufrió una notable caída en votos y escaños, de la que se deriva la dimisión de su líder, Albert Rivera. En la figura 3 se pueden observar algunos tuits de ejemplo que resumen la cobertura mediática de antes y después de estas elecciones generales.

Figura 3. Tuits representativos de antes y después de las elecciones del 10N

Antes de las elecciones
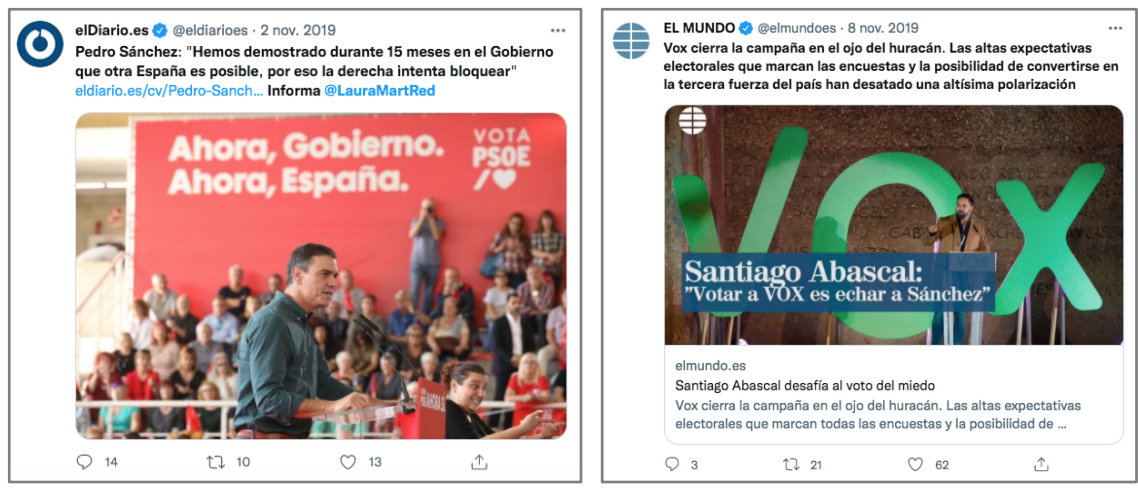

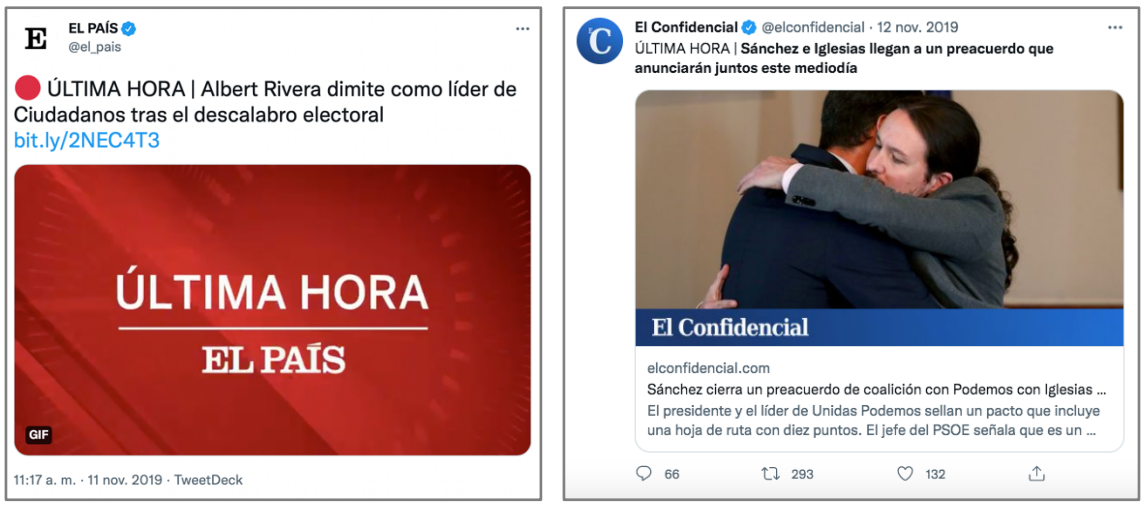

Fuente: elaboración propia.

A raíz de estas conclusiones conviene reflexionar sobre las observaciones realizadas. En primer lugar, es importante señalar que el interés por los temas políticos y por partidos y personajes concretos sigue patrones lógicos basados en el interés informativo que se asume en los días del estudio: antes de las elecciones, con los partidos llamados a ganar o crecer, y tras las elecciones, con aquellos que han tenido un desempeño sorprendente o que tienen posibilidades de formar gobierno. Es esperable que estos patrones sean semejantes en los contenidos publicados en las webs o diarios impresos de los medios analizados, por lo que futuros análisis comparativos entre el comportamiento de los medios en sus soportes originales y en sus redes sociales permitirán comprobar esta hipótesis. Igualmente, tampoco sorprende que los partidos que más interacciones despiertan sean los más polarizados y polarizadores (VOX y UP), aunque sí es curioso que las ideologías de los medios no jueguen un papel significativo en este aspecto - ni, en realidad, en ningún otro del estudio-. Esto podría derivarse del hecho de que las redes sociales permitan que los medios lleguen a públicos mucho más diversos de lo que lo hacen sus soportes originales; en este sentido, la teoría de Song, Gil de Zúñiga y Boomgaarden (2020) sobre el fenómeno news find me podría ayudar a explicar esta realidad. Más trabajo será necesario en este campo específico, pues esa semejanza en los patrones de interacción con medios de tan distinta ideología podría cuestionar en parte teorías como las cámaras de eco, reforzando debates sobre el rol de los medios sociales, entre quienes defienden que facilita la discusión y quienes considera que refuerza los propios puntos de vista (Moragas-Fernández, Grau-Masot y Capdevila-Gómez, 2019). 
Cabe señalar que este estudio se ha centrado en el uso de Twitter como herramienta de información política por parte de los principales medios de comunicación escrita y digital en España, poniendo de relieve la importancia de seguir profundizando en cómo los principales medios utilizan sus cuentas en medios sociales en un contexto en el que estas herramientas son esenciales como canal de información. Será útil, en este sentido, ampliar el estudio a redes sociales diferentes a Twitter, para comparar si las observaciones realizadas aquí son válidas en otras plataformas sociales.

Por último, conviene destacar, además, que existen limitaciones en el estudio, como la posibilidad de que cierta cantidad de tuits de contenido político que no hayan utilizado los términos de análisis descritos en la Metodología no hayan sido incluidos en el análisis. Igualmente, haber colocado el foco sobre los cinco partidos más relevantes de ámbito nacional ha podido limitar la capacidad de ofrecer resultados aplicables al resto de grupos con representación parlamentaria. Así, aunque un análisis exploratorio previo con una submuestra aleatoria mostró que el volumen de tuits que hayan podido quedar fuera del estudio por estos motivos es reducido, convendrá seguir realizando análisis que amplíen las observaciones realizadas en este.

\section{Referencias}

Arcila-Calderón, Carlos; Ortega-Mohedano, Félix; Amores, Javier J. y Trullenque, Sofía (2017). Análisis supervisado de sentimientos políticos en español: clasificación en tiempo real de tweets basada en aprendizaje automático. En: El profesional de la información, Vol. 26, n ${ }^{\circ}$ 5. Barcelona: EPI, 973-982.

Blanco-Herrero, David; Frías-Vázquez, Maximiliano; Sánchez-Holgado, Patricia y Amores, Javier J. (2020). Análisis de los perfiles de los principales medios de comunicación españoles durante la campaña del 10N. En J. Cruz (Coord.) El apagón analógico y el despertar del periodismo digital. Ecuador: Pontificia Universidad Católica del Ecuador.

Bruns, Axel; Burgess, Jean (2012). Researching news discussion on Twitter. En: Journalism Studies, Vol. 13, $n^{o}$ 5-6. Estados Unidos: Taylor and Francis, 801-814. Calvo-Rubio, Luis Mauricio (2017). El uso de Twitter por los partidos políticos durante la campaña del 20D. En: Sphera Publica, Vol. 1, no 17. Murcia: Universidad Católica San Antonio de Murcia, 111-131.

Campos-Domínguez, Eva (2017). Twitter y la comunicación política. En: El profesional de la información, Vol. 26, $n^{o}$ 5. Barcelona: EPI, 785-793.

Carlson, Matt (2018). Facebook in the News. En: Digital Journalism, Vol. 6, $\mathrm{n}^{\mathrm{o}} 1$. Reino Unido: Taylor and Francis, 4-20. 
Casero-Ripollés, Andreu (2018). Investigación sobre información política y redes sociales: puntos clave y retos de futuro. Profesional de la información, Vol. 27, $\mathrm{n}^{\mathrm{o}}$ 5. Barcelona: EPI, 964-974.

Castells, Manuel (2010). Comunicación y poder. Madrid: Alianza Editorial.

Conway, Bethany A.; Kenski, Kate y Wang. Di (2015). The rise of Twitter in the political campaign: Searching for intermedia agenda-setting effects in the presidential primary. En: Journal of Computer-Mediated Communication, Vol. 20, $\mathrm{n}^{\mathrm{o}}$ 4. Estados Unidos, Wiley, 363-380.

D'heer, Evelien; Verdegem, Pieter (2014). Conversations about the elections on Twitter: Towards a structural understanding of Twitter's relation with the political and the media field. En: European Journal of Communication, Vol. 29, $\mathrm{n}^{\circ} 6$. Reino Unido: SAGE, 720-734.

Fenoll, Vicente; Cárcamo-Ulloa, Luis; Sáez-Trumper, Diego (2018). The usage of Twitter from the Spanish media during the elections. En: Estudios sobre el Mensaje Periodístico, Vol. 24, $\mathrm{n}^{\circ}$ 2. Madrid: Universidad Complutense de Madrid, 1223-1238.

García-De-Torres, Elvira; Yezers'Ka, Lyudmyla; Rost, Alejandro; Calderín, Mabel; Edo, Concha; Rojano, Miladys; Said, Elías; Jerónimo, Pedro; Arcila, Carlos; Serrano, Ana; Sánchez, Jorge; Corredoira, Loreto (2011). Uso de Twitter y Facebook por los medios iberoamericanos. En: El profesional de la información, Vol. 20, n ${ }^{0}$ 6. Barcelona: EPI 611-620.

García-Ortega, Carmela; Zugasti-Azagra, Ricardo (2018). Gestión de la campaña de las elecciones generales de 2016 en las cuentas de Twitter de los candidatos: entre la autorreferencialidad y la hibridación mediática. En: El profesional de la información, Vol. 27, nº 6 . Barcelona: EPI, 1215-1224.

Gil-Ramírez, Marta; Gómez de Travesedo-Rojas, Ruth (2021). Repensar la comunicación política en el contexto digital. Ciberdemocracia ¿democracia deliberativa? En: Vivat Academia, n ${ }^{0}$ 154. Madrid: Fórum, 63-87.

González Molina, Sonia y Ramos del Cano, Fátima (2014). Las redes sociales en el ámbito periodístico: ¿cómo usan los medios europeos de referencia sus perfiles en Twitter y Facebook? En: Comunicación y Hombre, Vol. 10. Madrid: Universidad Francisco de Vitoria, 37-52.

Groshek, Jakob; Al-Rawi, Ahmed (2013). Public sentiment and critical framing in social media content during the 2012 US presidential campaign. En: Social Science Computer Review, Vol. 31, nº 5. Estados Unidos: SAGE, 563-576.

Hermida, Alfred (2013). \#Journalism. Reconfiguring Journalism Research about Twitter One Tweet at a Time. En: Digital Journalism, Vol. 1, $\mathrm{n}^{\circ}$ 3. Reino Unido: Taylor and Francis, 295-313. 
Hermida, Alfred; Mellado, Claudia (2020). Dimensions of Social Media Logics: Mapping Forms of Journalistic Norms and Practices on Twitter and Instagram. En: Digital Journalism, Vol. 8, $\mathrm{n}^{\circ}$ 7. Londres: Taylor and Francis, 864-884.

Houston, J. Brian; McKinney, Mitchell, S.; Thorson, Esther; Hawthorne, Joshua; Wolfgang, J. David; Swasy, Alecia (2018). The twitterization of journalism: User perceptions of news tweets. En: Journalism, Vol. 21, $\mathrm{n}^{0}$ 5. Reino Unido: SAGE, 614-632.

Jivkova-Semova, Dimitrina; Requeijo-Rey, Paula; Padilla-Castillo, Graciela (2017). Usos y tendencias de Twitter en la campaña a elecciones generales españolas del 20D de 2015: hashtags que fueron trending topic. En: El profesional de la información, Vol. 26, $\mathrm{n}^{\circ}$ 5. Barcelona: EPI, 824-837.

Justel-Vázquez, Santiago; Fernández-Planells, Ariadna; Victoria-Mas, María y Lacasa-Mas, Iván (2018). Twitter e información política en la prensa digital: la red social como fuente de declaraciones en la era Trump. Profesional de la Información, Vol. 27, n 5 . Barcelona: EPI, 984-993.

Lee, Jayeon; Xu, Weiai (2018). The more attacks, the more retweets: Trump's and Clinton's agenda setting on Twitter. En: Public Relations Review, Vol. 44, $\mathrm{n}^{0} 2$. Países Bajos: Elsevier, 201-213.

López-García., Guillermo (2016). 'New' vs 'old' leaderships: the campaign of Spanish general elections 2015 on Twitter. En: Communication y Society, Vol. 29, $\mathrm{n}^{\mathrm{o}}$ 3. Pamplona: Universidad de Navarra,149-168.

López Meri, Amparo (2015a). Twitter como fuente informativa de sucesos imprevistos: el seguimiento de hashtags en el caso \#ArdeValencia. En: Disertaciones: Anuario electrónico de estudios en Comunicación Social, Vol. 8, $\mathrm{n}^{\mathrm{0}}$ 1. Rosario: Universidad del Rosario, 27-51.

López Meri, Amparo (2015b). Redes sociales y campañas electorales: Twitter como fuente informativa en las elecciones catalanas del\# 25N. En: Comunicació: revista de recerca i d'anàlisi, Vol. $32, \mathrm{n}^{\circ} 2$. Barcelona: Societat Catalana de Comunicació, 115-137.

López Meri, Amparo; Marcos-García, Silvia; Casero-Ripollés, Andreu (2017). ¿Qué hacen los políticos en Twitter? Funciones y estrategias comunicativas en la campaña electoral española de 2016. En: El profesional de la información, Vol. 26, $\mathrm{n}^{\mathrm{o}}$ 5. Barcelona: EPI, 795-804.

Marcos-García, Silvia; Alonso-Muñoz, Alonso; López Meri, Amparo (2021). Campañas electorales y Twitter. La difusión de contenidos mediáticos en el entorno digital. En: Cuadernos.Info, $\mathrm{n}^{0}$ 48. Chile: Pontificia Universidad Católica de Chile, 27-49.

Marín Dueñas, Pedro Pablo; Díaz Guerra, Aurora (2016). Uso de Twitter por los partidos y candidatos políticos en las elecciones autonómicas de Madrid 2015. En: 
Ámbitos: Revista Internacional de Comunicación, Vol. 32. Sevilla: Universidad de Sevilla, 1-15.

McElroy, Kathleen (2013). Where Old (Gatekeepers) Meets New (Media). En: Journalism Practice, Vol. 7, $\mathrm{n}^{\circ}$ 6. Reino Unido: Tayloer and Francis, 755-771.

McCombs, Maxwell E; Shaw, Donald L. (1972). The agenda-setting function of mass media. En: Public opinion quarterly, Vol. $36, \mathrm{n}^{\circ}$ 2. Washington, EEUU: American Association for Public Opinion Research, 176-187.

Moragas-Fernández, Carlota M.; Grau-Masot, Josep-María y Capdevila-Gómez, Arantxa (2019). Articulación de la influencia en Twitter ante el anuncio de la Ley del referéndum en Cataluña. En: Profesional De La Información, Vol. 28, $\mathrm{n}^{\circ} 3$. Barcelona: EPI SCP, e280320.

Newman, Nic; Fletcher, Richard; Kalogeropoulos, Antonis y Nielsen, Rasmus (2019). Reuters Institute Digital News Report 2019. Oxford: Reuters Institute for the Study of Journalism.

Noguera, José Manuel (2010). Redes sociales como paradigma periodístico. Medios españoles en Facebook. En: Revista Latina de Comunicación Social, Vol. 65. La Laguna: Sociedad Latina de Comunicación Social, 176-186.

Quevedo Redondo, Raquel; Portalés-Oliva, Marta y Berrocal Gonzalo, Salomé (2016). The image use on Twitter during the 2015 municipal election campaign in Spain. En: Revista Latina de Comunicación Social, Vol. 71. La Laguna: Sociedad Latina de Comunicación Social, 85-107.

Requejo Alemán, José Luis y Herrera Damas, Susana (2011). Difundir información, principal uso de los diarios generalistas españoles en Twitter. En: Textual y Visual Media, Vol. 4. Madrid: Sociedad Española de Periodística, 149-182.

Rodríguez, Roberto; Ureña, Daniel (2011). Diez razones para el uso de Twitter como herramienta en la comunicación política y electoral. En: Comunicación y pluralismo, Vol. 10. Salamanca: Universidad Pontificia de Salamanca, 89-116.

Sáez-Trumper, Diego (2011). La información en Internet: Breve estado del arte para discutir el poder de los usuarios v/s los medios tradicionales de comunicación en la red. En: Revista Austral Ciencias Sociales, Vol. 20. Chile: Universidad Austral de Chile, 71-79.

Skogerbø, Eli y Krumsvik, Arne H. (2015). Newspapers, Facebook and Twitter. Intermedial agenda setting in local election campaigns. En: Journalism Practice, Vol. 9, $n^{\circ}$ 3. Londres: Taylor and Francis, 350-366.

Song, Hyunjin; Gil de Zúñiga, Homero y Boomgaarden, Hajo G. (2020). Social Media News Use and Political Cynicism: Differential Pathways Through "News Finds Me” Perception. En: Mass Communication and Society, Vol. 23, $\mathrm{n}^{\circ} 1$. Londres: Taylor and Francis, 47-70. 
Suárez, Juan Carlos y Cruz, Jesús (2016). Los dilemas deontológicos del uso de las redes sociales como fuentes de información. Análisis de la opinión de los periodistas de tres países. En: Revista Latina de Comunicación Social, Vol. 71. La Laguna: Sociedad Latina de Comunicación Social, 66-84.

Suau-Gomila, Guillem (2020). Microblogging electoral: la estrategia comunicativa de Podemos y Ciudadanos en Twitter en las campañas electorales del 20D y el 26J. En: Prisma Social, ${ }^{0} 28$. Madrid: Fundación iS+D para la investigación Social Avanzada, 103-126.

Turnbull-Dugarte, Stuart; Rama, José y Santana, Andrés (2020). The Baskerville's dog suddenly started barking: voting for VOX in the 2019 Spanish general elections. En: Political Research Exchange, Vol. 2, $\mathrm{n}^{\circ}$ 1. Reino Unido: Taylor and Francis, 1781543. 